Published in final edited form as:

Support Care Cancer. 2015 July ; 23(7): 2019-2024. doi:10.1007/s00520-014-2556-x.

\title{
Stopping paclitaxel premedication after two doses in patients not experiencing a previous infusion hypersensitivity reaction
}

\author{
Michael J. Berger, \\ Pharmacy Department, The Stefanie Spielman Comprehensive Breast Center, The James \\ Cancer Hospital and Solove Research Institute at the Ohio State University, Columbus, USA \\ Specialty Practice Pharmacist, Department of Pharmacy, The Stefanie Spielman Comprehensive \\ Breast Center, 1145 Olentangy River Rd, Columbus, OH 43210, USA \\ Craig Vargo, \\ Pharmacy Department, The Stefanie Spielman Comprehensive Breast Center, The James \\ Cancer Hospital and Solove Research Institute at the Ohio State University, Columbus, USA

\section{Mary Vincent,} \\ Pharmacy Department, The Stefanie Spielman Comprehensive Breast Center, The James \\ Cancer Hospital and Solove Research Institute at the Ohio State University, Columbus, USA

\section{Katy Shaver,} \\ Pharmacy Department, The Stefanie Spielman Comprehensive Breast Center, The James \\ Cancer Hospital and Solove Research Institute at the Ohio State University, Columbus, USA

\section{Gary Phillips,} \\ The Ohio State University Center for Biostatistics, Columbus, USA
}

\section{Rachel Layman,}

Breast Program, The Stefanie Spielman Comprehensive Breast Center, The James Cancer Hospital and Solove Research Institute at the Ohio State University, Columbus, USA

\section{Erin Macrae,}

Breast Program, The Stefanie Spielman Comprehensive Breast Center, The James Cancer Hospital and Solove Research Institute at the Ohio State University, Columbus, USA

\section{Ewa Mrozek,}

Breast Program, The Stefanie Spielman Comprehensive Breast Center, The James Cancer Hospital and Solove Research Institute at the Ohio State University, Columbus, USA

\section{Bhuvaneswari Ramaswamy,}

Breast Program, The Stefanie Spielman Comprehensive Breast Center, The James Cancer Hospital and Solove Research Institute at the Ohio State University, Columbus, USA

Robert Wesolowski,

Correspondence to: Michael J. Berger, mi chael . bergereosumc . edu.

Disclaimers None.

Previous publication of manuscript None. 
Breast Program, The Stefanie Spielman Comprehensive Breast Center, The James Cancer Hospital and Solove Research Institute at the Ohio State University, Columbus, USA

Charles L. Shapiro, and

Breast Program, The Stefanie Spielman Comprehensive Breast Center, The James Cancer Hospital and Solove Research Institute at the Ohio State University, Columbus, USA

\section{Maryam B. Lustberg}

Breast Program, The Stefanie Spielman Comprehensive Breast Center, The James Cancer Hospital and Solove Research Institute at the Ohio State University, Columbus, USA

Michael J. Berger: michael.berger@osumc.edu

\section{Abstract}

Purpose-Paclitaxel-based chemotherapy continues to be an integral component of breast cancer treatment. Prolonged use of paclitaxel may result in repeated doses of premedications that can have unwanted side effects. Infusion hypersensitivity reactions occurring beyond the second dose of paclitaxel are infrequent and not well characterized. We previously published the results of a small, prospective pilot trial demonstrating the safety and feasibility of discontinuing premedications in patients who received the first two doses of paclitaxel-based chemotherapy without experiencing an infusion hypersensitivity reaction. In this study, we aimed to retrospectively characterize the incidence of rescue medication using this abbreviated premedication regimen in our institution following the publication of the pilot study.

Methods-Patients with stages I-IV breast cancer who received paclitaxel from January 2011 through June 2013 were screened for eligibility. Patients who did not experience an infusion hypersensitivity reaction with their first or second dose of paclitaxel and discontinued paclitaxel premedication for subsequent doses were included in this analysis. The primary endpoint was to estimate the incidence of rescue medication use for the treatment of paclitaxel infusion hypersensitivity during doses three to six of paclitaxel in the study population.

Results-In total, 449 patients received paclitaxel-based chemotherapy for the treatment of breast cancer during the interval time period. After receiving the first two doses of paclitaxelbased chemotherapy without experiencing an infusion hypersensitivity reaction, 234 breast cancer patients had their premedications discontinued for all remaining paclitaxel doses. These patients tolerated future paclitaxel doses without severe or life-threatening complications related to infusion hypersensitivity. The majority of patients did not have any symptoms of an infusion reaction, with only two of these patients requiring rescue medication to treat an infusion hypersensitivity reaction with subsequent paclitaxel doses $(0.85 ; 95 \%$ confidence interval (CI), $0.10-3.05 \%)$.

Conclusions-Discontinuation of paclitaxel premedications in breast cancer patients who have not experienced an infusion hypersensitivity reaction with the first two doses of paclitaxel is not associated with increased rate of rescue medication use for infusion hypersensitivity.

\section{Keywords}

Abbreviated; Paclitaxel; Premedication; Prophylaxis; Hypersensitivity 


\section{Introduction}

Due to its hydrophobic properties, paclitaxel must be emulsified in a vehicle consisting of $50 \%$ polyoxyethylated castor oil (Cremophor EL) and 50\% ethanol. Despite premedication with a corticosteroid and both histamine- 1 and histamine- 2 receptor antagonists, hypersensitivity reactions (all grades) during intravenous administration of paclitaxel for the treatment of solid tumors in clinical studies occurred in $41 \%$ of patients, and "severe" (grade 3 or higher) hypersensitivity reactions occurred in $2 \%$ of patients [1]. Studies using paclitaxel for the treatment of breast cancer at doses between 80 and $90 \mathrm{mg} / \mathrm{m}^{2}$ given as a 1$\mathrm{h}$ infusion report a similar incidence (less than $3 \%$ ) of grade 3 or higher hypersensitivity reactions $[2,3]$.

Previous studies have demonstrated that a simplified, intravenous premedication regimen consisting of dexamethasone and both histamine- 1 and histamine-2 receptor antagonists administered 30 min prior to paclitaxel infusion can successfully reduce the incidence and severity of hypersensitivity reactions with comparable clinical efficacy to older regimens that employed dexamethasone $20 \mathrm{mg}$ given orally 12 and $6 \mathrm{~h}$ and immediately prior to paclitaxel, although some experts remain critical of this simplified approach [4-6]. The most frequently used corticosteroid in the prevention of paclitaxel-induced hypersensitivity reactions is dexamethasone, which is among the most potent and longest acting of the corticosteroids. With prolonged use of paclitaxel, especially when given in a once-weekly fashion, patients are exposed to repeated doses of dexamethasone. Significant side effects associated with short-term and prolonged use of corticosteroids are well documented, including hyperglycemia, insomnia, gastritis, fluid retention, weight gain, immune suppression, acne, skin changes, cataracts, osteoporosis, mental status changes, and adrenal suppression. In addition, when administered as a rapid intravenous bolus, dexamethasone has been associated with perianal pruritus. Diphenhydramine, the most commonly employed histamine-1 receptor antagonist for the prevention of paclitaxel-induced hypersensitivity, is commonly associated with drowsiness and can paradoxically cause dystonia and restlessness when $50 \mathrm{mg}$ is administered intravenously [7].

To minimize the adverse effects of corticosteroids, several strategies exploring an alternative paclitaxel premedication regimen have been published including incorporation of a paclitaxel test dose, dexamethasone dose reduction, or use of a dexamethasone tapering schedule [8-11]. In an attempt to further simplify paclitaxel premedication and avoid unnecessary side effects caused by premedication regimens, we conducted a prospective pilot trial and reported the results; patients who had received their first two doses of paclitaxel without experiencing an infusion hypersensitivity reaction had their paclitaxel premedications discontinued for future paclitaxel doses. Fifty-five patients were included in the primary analysis; none of these patients required parenteral rescue medication with subsequent paclitaxel doses, and no infusion hypersensitivity reactions (any grade) were observed. The incidence of parenteral rescue medication in this study was $0.0 \%$ with an exact one-sided binomial $95 \%$ confidence interval of $0.0-5.3 \%$ [12]. This was in contrast to standard paclitaxel premedication regimens in which patients may continue to receive premedications prior to each dose of paclitaxel, even if they had not experienced a previous infusion hypersensitivity reaction. 
Since the publication of the pilot study, our breast medical oncology department has administered paclitaxel to hundreds of patients and successfully discontinued standard premedications after the second paclitaxel dose in many of these patients. Although the pilot trial was compelling and provided evidence for safety and feasibility for the discontinuation of paclitaxel premedications after two doses of paclitaxel in the absence of infusion hypersensitivity, the publication did not result in a uniform practice change. Therefore, local practice varied, as some providers would stop premedications after the second or later doses, and other providers would not stop premedications at all unless the patient was experiencing side effects from the premedications themselves. This retrospective study sought to estimate the incidence of rescue medication usage during paclitaxel doses three through six for patients who had an abbreviated premedication regimen.

\section{Materials and methods}

\section{Patients}

The research protocol was approved by the Institutional Review Board. Eligibility criteria included the following: patients $\geq 18$ years old who received at least four doses of paclitaxel as a single agent or in combination (excluding cisplatin or carboplatin) for the treatment of any stage breast cancer. Exclusion criteria included patients receiving daily doses of systemic corticosteroids; patients receiving paclitaxel albumin-bound; patients who had received at least one previous dose of paclitaxel, docetaxel, or paclitaxel albumin-bound within the last 12 months; patients receiving paclitaxel in combination with carboplatin or cisplatin (due to risk of hypersensitivity with platinum compounds); patients who never discontinued premedications; patients who did not receive at least two doses of paclitaxel with required premedication; patients who had their premedications discontinued after the second paclitaxel dose but did not receive at least two subsequent paclitaxel doses without premedication; patients who switched to a different taxane before receiving four doses of paclitaxel. Patients were screened for eligibility using our electronic medical record (EPIC/ BEACONTM) database. Data was collected on all eligible patients who received paclitaxel at The Stefanie Spielman Comprehensive Breast Center (SSCBC) at The Ohio State University from January 1, 2011 through June 30, 2013.

\section{Treatment}

Eligible patients who received paclitaxel-based chemotherapy had standard paclitaxel premedications (dexamethasone $20 \mathrm{mg}$ IVP, diphenhydramine $50 \mathrm{mg}$ IVP, and famotidine $20 \mathrm{mg}$ IVP) administered at least $30 \mathrm{~min}$ prior to the start of paclitaxel. If patients had not experienced a hypersensitivity reaction (any grade) during the first two paclitaxel infusions, dexamethasone, diphenhydramine, and famotidine were discontinued prior to the third dose and for all subsequent paclitaxel doses.

\section{Endpoints and assessment}

The primary endpoint was to estimate the incidence of rescue medication usage during paclitaxel doses three through six for patients whose premedication regimen was discontinued after the first two doses of paclitaxel. 
Clinical signs and symptoms of paclitaxel hypersensitivity reactions may vary (see Table 1). Grading of these hypersensitivity reactions is challenging as no standard criteria is allencompassing, and in routine clinical practice, parenteral "rescue" medications are often administered at the onset of the infusion hypersensitivity reaction to minimize the severity and progression of the reaction. We selected the use of rescue medication as our primary endpoint, rather than occurrence of an infusion hypersensitivity reaction, because the former is a more measurable and objective endpoint given the difficulty of grading these reactions.

The use of rescue medication for the treatment of paclitaxel infusion hypersensitivity was defined as the administration of at least one parenteral rescue medication (excluding $0.9 \%$ sodium chloride) including hydrocortisone, dexamethasone, diphenhydramine, famotidine, or epinephrine. Each patient's electronic chemotherapy order contained standing orders enabling the chemotherapy nurse to identify and treat a hypersensitivity reaction using a standard rescue medication protocol. Eligible patients had data recorded for up to six doses of paclitaxel, after which time the incidence of infusion hypersensitivity reactions would be increasingly rare.

\section{Statistical analysis}

This single arm retrospective study explored the incidence of rescue medication required between doses 3 and 6; this was estimated and the associated 95\% confidence interval (CI) was generated using exact binomial methods. Categorical patient demographics and clinical characteristics are described using frequencies and percentages, while age and weight are described using mean and standard deviation. No inferential testing was done to compare this incidence rate to the earlier prospective study as this current study is only descriptive in nature.

We expected the incidence of rescue medication to be less than $5 \%$, thus if $9(4.5 \%)$ patients out of 200 required rescue medication, then $95 \%$ CI would have been $2.1-8.4 \%$. The incidence and the width of the confidence interval $(6.3 \%)$ would be narrow enough to verify our published findings from the earlier prospective pilot trial where 0 out of 55 subjects required rescue medication.

\section{Results}

In total, 449 patients received paclitaxel-based chemotherapy for the treatment of breast cancer at the SSCBC. After receiving the first two doses of paclitaxel-based chemotherapy without experiencing an infusion hypersensitivity reaction (any grade), 234 breast cancer patients $(52 \%)$ met all eligibility criteria and had their premedications discontinued for all remaining paclitaxel doses. Baseline characteristics, including the 215 patients $(47 \%)$ who did not meet eligibility criteria, are summarized in Table 2 , and type of paclitaxel-based chemotherapy regimens received is summarized in Table 3. All but two were female, $70 \%$ $(n=164)$ had at least one chronic medical condition, and $12 \%(n=28)$ were diagnosed with stage IV breast cancer. There were no significant differences in age, weight, or chronic medical conditions between these groups. A higher percentage of patients in the excluded group had stage IV disease versus the included group (20.6 versus $12.0 \%$, respectively, $p=$ 0.015). Reasons for ineligibility are summarized in Table 4 . Of these 215 excluded patients, 
$143(66.5 \%)$ were excluded because they never discontinued premedication; 31 patients (14.4\%) who had paclitaxel premedications safely discontinued after the second dose but requested to resume them with later doses in hopes of alleviating non-infusion-related toxicities possibly attributed to paclitaxel; rash $(n=11)$, edema $(n=5)$, arthralgias/pain $(n=$ $3)$, nausea $(n=3)$, urticaria $(n=2)$, and non-specific patient request/other $(n=7) ; 20(9.3 \%)$ patients were receiving concurrent carboplatin.

Two hundred thirty-four patients met inclusion criteria and were included in the primary analysis. Of those, two subjects $(0.85 ; 95 \% \mathrm{CI}, 0.10-3.05 \%)$ required rescue medications to treat a paclitaxel infusion hypersensitivity reaction between doses 3 and 6 .

After discontinuing premedications following her second paclitaxel dose, subject 1 received her third and fourth doses of paclitaxel without incidence. During her fifth dose of paclitaxel, approximately 60 min into a 60-min infusion, the patient reported flushing, throat tightness, and nausea. The paclitaxel was stopped, hydrocortisone $50 \mathrm{mg}$ IVP was administered, and her symptoms were completely resolved about $1 \mathrm{~h}$ later. Her vital signs remained normal throughout this time period. The patient resumed premedications prior to future paclitaxel doses and received six more doses of paclitaxel at the normal infusion rate without further incidence of hypersensitivity. This hypersensitivity reaction was somewhat atypical as the reaction occurred at the conclusion rather than the beginning of the infusion.

After discontinuing premedications following her second paclitaxel dose, subject two developed flushing, lower back pain, cough, and chest tightness approximately 6 min into a 60-min infusion with her third paclitaxel dose. The paclitaxel was stopped; hydrocortisone $50 \mathrm{mg}$ IVP and diphenhydramine $25 \mathrm{mg}$ IVP were administered. The patient experienced transient grade 1 hypertension. Her vital signs returned to baseline within $45 \mathrm{~min}$, and the paclitaxel infusion was resumed at 50\% rate for $15 \mathrm{~min}$ and then re-escalated to goal rate for the remainder of the infusion, which the patient tolerated without further hypersensitivity. Unfortunately, the patient was found to have disease progression and did not receive further paclitaxel doses.

\section{Discussion}

The exact mechanism of paclitaxel or Cremophor EL-induced hypersensitivity is unknown, although histamine release by mast cells and basophils are thought to be partially responsible. As these reactions may occur with the first exposure, an IgE-mediated reaction is unlikely. Paclitaxel infusion hypersensitivity reactions are well characterized; regardless of paclitaxel dose or infusion rate, up to $95 \%$ of hypersensitivity reactions occur during the first or second dose, and in almost all cases, the hypersensitivity reaction develops within the first 5-10 min of the infusion [13,14]. The overwhelming majority of patients who experience an initial hypersensitivity reaction can successfully resume and complete paclitaxel treatment [9]. In a pooled toxicity analysis of over 800 patients receiving paclitaxel as a single agent for the treatment of solid tumors, no grade 3 or higher paclitaxel infusion hypersensitivity reactions occurred beyond the third dose [3]. Other investigators observed two patients (5\%) who experienced a "clinically relevant" initial hypersensitivity infusion reaction occurring beyond the second dose [13]. We are unable to compare the 
results of our primary endpoint (two patients requiring rescue medication after premedications were discontinued) to historical controls since the true incidence of rescue medication usage after the second dose is not characterized in the literature.

Interestingly, in our report, there was a subset of 31 patients excluded from the primary analysis who despite having no infusion hypersensitivity reactions felt that non-infusionrelated and late side effects possibly attributed to paclitaxel (such as rash, edema, urticaria, nausea, arthralgias, and other non-specific reasons) could be ameliorated by premedications and thus requested that they be resumed after they were previously discontinued.

Due to practice variations between providers or per patient request, premedications were occasionally discontinued after the third or fourth paclitaxel doses $(n=9)$. These patients were excluded from the primary analysis, although during data collection, it was noted that two of these patients required rescue medication for infusion hypersensitivity reactions after premedications had been stopped and are therefore worth discussing here.

After discontinuing premedications following her third paclitaxel dose, subject 1 received her fourth dose of paclitaxel without incidence. During her fifth dose of paclitaxel, approximately 4 min into a 60-min infusion, the patient reported a feeling of warmth, flushing, and dyspnea. The paclitaxel was stopped, $0.9 \%$ sodium chloride was infused wide open to gravity, and hydrocortisone $50 \mathrm{mg}$ IVP was administered. Her symptoms resolved over the next $30 \mathrm{~min}$. Her vital signs remained normal throughout this time period. The patient resumed the paclitaxel infusion (now $35 \mathrm{~min}$ after stopping previously) at 50\% infusion rate for $10 \mathrm{~min}$ and then escalated to the goal rate for the remainder of the infusion which the patient tolerated without further hypersensitivity. With her sixth dose of paclitaxel, premedications were resumed-but approximately 9 min into a 60-min infusion, the patient again reported warmth, flushing, and dyspnea. Rescue medications were given, symptoms resolved, and her vital signs remained stable throughout. The infusion was resumed and escalated to goal rate without further hypersensitivity. With her seventh dose, in addition to paclitaxel premedications, her paclitaxel tubing was primed with active drug, and the infusion was given as a four-step rate escalation, starting at $15 \%$ of goal rate for 10 $\mathrm{min}$, then increasing to $30 \%$ of goal rate for $10 \mathrm{~min}$, then increasing to $50 \%$ of goal rate for $10 \mathrm{~min}$, then increasing to goal rate [15]. The patient tolerated this 90-min infusion without incidence of hypersensitivity and received four more doses of paclitaxel in the future without incidence of hypersensitivity using this same strategy.

After discontinuing premedications following her third paclitaxel dose, during her fourth dose of paclitaxel, approximately $10 \mathrm{~min}$ into a 60-min infusion, subject two reported dyspnea and shaking. The chemotherapy nurse noted increased blood pressure and heart rate and decreased oxygen saturation. The paclitaxel was stopped, $0.9 \%$ sodium chloride was infused wide open to gravity, oxygen $2 \mathrm{l} / \mathrm{min}$ was started, and dexamethasone $20 \mathrm{mg} \mathrm{IVP,}$ diphenhydramine $50 \mathrm{mg}$ IVP, and famotidine $20 \mathrm{mg}$ IVP were administered. Her symptoms and vital signs returned to baseline over the next $60 \mathrm{~min}$. The patient resumed the paclitaxel infusion (now, 60 min after stopping previously) at goal rate for the remainder of the infusion which the patient tolerated without further hypersensitivity. With her fifth dose of paclitaxel, premedications were resumed-but approximately 9 min into a 60-min infusion, 
the patient reported cough and dyspnea. The paclitaxel was stopped, $0.9 \%$ sodium chloride was infused wide open to gravity, and hydrocortisone $50 \mathrm{mg}$ IVP was administered. Her vital signs and oxygen saturation were unchanged, and her symptoms resolved over the next $30 \mathrm{~min}$. The patient resumed the paclitaxel infusion (now $30 \mathrm{~min}$ after stopping previously) at $50 \%$ rate for $15 \mathrm{~min}$ and then increased to goal rate for the remainder of the infusion which the patient tolerated without further hypersensitivity. With her sixth dose, in addition to paclitaxel premedications, her paclitaxel tubing was primed with active drug, and the infusion was given as a four-step rate escalation, starting at $15 \%$ of goal rate for $10 \mathrm{~min}$, then increasing to $30 \%$ of goal rate for $10 \mathrm{~min}$, then increasing to $50 \%$ of goal rate for 10 $\mathrm{min}$, then increasing to goal rate. The patient tolerated this $90 \mathrm{~min}$ infusion without incidence of hypersensitivity and received 14 more doses of paclitaxel in the future without incidence of hypersensitivity using this same strategy.

With such a small number of patients (two in primary analysis and two more in the excluded group) who experienced a paclitaxel infusion hypersensitivity reaction after previously stopping premedications, it is difficult to draw comparisons between these patients or identify a predictive factor that predisposed them to a subsequent reaction. Two of these patients were receiving a weekly paclitaxel regimen dosed at $80 \mathrm{mg} / \mathrm{m}^{2}$ and two of them were receiving a weekly paclitaxel regimen dosed at $80 \mathrm{mg} / \mathrm{m}^{2}$ in combination with trastuzumab. Three of these patients had stage IV disease.

For patients who experience a severe paclitaxel hypersensitivity reaction, a rapid densensitization infusion for subsequent exposures may be considered. This approach utilizes a gradual increase in drug dosage during infusion, thus rendering mast cells and basophils temporarily unresponsive, allowing the patient to develop a transient tolerance to paclitaxel [15]. For mild or moderate paclitaxel hypersensitivity reactions, shortened versions of this desensitization protocol are often employed.

There are several limitations to this report including the retrospective nature and lack of a control arm. Quality of life with or without premedication administration, nursing convenience, and patient time spent in the infusion suite and cost data, collected before and after paclitaxel premedication discontinuation, also would have been of interest. Despite a large number of patients who were ineligible and therefore excluded from primary analysis because their premedications were never discontinued due to their provider's practice pattern $(n=143)$, selection bias seems unlikely as this was a retrospective study, and there were no significant demographic differences between the included and excluded groups. A small number $(13.2 \%)$ of patients included in the primary analysis received $175 \mathrm{mg} / \mathrm{m}^{2}$ doses, and although our study was not powered to look at differences between paclitaxel doses, this approach appears to be applicable regardless of paclitaxel dose.

As the usage of weekly paclitaxel administration for the treatment of breast cancer has increased, the necessity for repeated doses of premedications to prevent infusion hypersensitivity reactions with every dose and the safety of discontinuing these premedications are relevant questions. The data reported here strengthens our previous conclusions and supports this simplified approach of stopping premedications after two doses. This approach looks promising but must be validated with a larger, prospective, and 
randomized trial. Subsequent prospective trials should also include cost analysis as well as validated questionnaires to assess the side effects of standard premedications and the degree of interference that these symptoms cause on quality of life.

\section{Acknowledgments}

Research funding None.

\section{References}

1. Taxol ${ }^{\circledR}$ injection (package insert). Princeton, NJ: Bristol-Myers Squibb Company; 2007 Jul.

2. Sparano JA, et al. Weekly paclitaxel in the adjuvant treatment of breast cancer. N Engl J Med. 2008; 358(16):1663-1671. [PubMed: 18420499]

3. Miller K, et al. Paclitaxel plus bevacizumab versus paclitaxel alone for metastatic breast cancer. $\mathrm{N}$ Engl J Med. 2007; 357(26):2666-2676. [PubMed: 18160686]

4. Markman M, et al. An effective and more convenient drug regimen for prophylaxis against paclitaxel-associated hypersensitivity reactions. J Cancer Res Clin Oncol. 1999; 125(7):427-429. [PubMed: 10394964]

5. Bookman MA, et al. Intravenous prophylaxis for paclitaxel-related hypersensitivity reactions. Semin Oncol. 1997; 24(6 Suppl 19):S19-13-S19-15. [PubMed: 9427258]

6 . Kloover JS, et al. Fatal outcome of a hypersensitivity reaction to paclitaxel: a critical review of premedication regimens. Br J of Cancer. 2004; 90:304-305. [PubMed: 14974481]

7. Micromedex. Diphenhydramine. v 1.0. 2007 Available at: http://www.thomsonhc.com/ micromedex $2 /$ librarian.

8. Quock J, et al. Premedication strategy for weekly paclitaxel. Cancer Invest. 2002; 20(5-6):666-672. [PubMed: 12197222]

9. Koppler, H., et al. Onkologie. Vol. 24. German: English; 2001. Dose reduction of steroid premedication for paclitaxel: no increase of hypersensitivity reactions; p. 283-285.

10. Braverman AS, et al. Tapering and discontinuation of glucocorticoid prophylaxis during prolonged weekly to biweekly paclitaxel administration. Chemotherapy. 2005; 51(2-3):116-119. [PubMed: 15886470]

11. Lenz HJ. Management and preparedness for infusion and hypersensitivity reactions. Oncologist. 2007; 12(5):601-609. [PubMed: 17522249]

12. Berger MJ, et al. Feasibility of stopping paclitaxel premedication after two doses in patients not experiencing a previous infusion hypersensitivity reaction. Support Care Cancer. 2012; 20(9): 1991-1997. [PubMed: 22089428]

13. Markman M, et al. Paclitaxel-associated hypersensitivity reactions: experience of the gynecologic oncology program of the Cleveland Clinic Cancer Center. J Clin Oncol. 2000; 18(1):102-105. [PubMed: 10623699]

14. Zanotti KM, Markman M. Prevention and management of antineoplastic-induced hypersensitivity reactions. Drug Saf. 2001; 24(10):767-779. [PubMed: 11676304]

15. Feldweg AM, et al. Rapid desensitization for hypersensitivity reactions to paclitaxel and docetaxel: a new standard protocol used in 77 successful treatments. Gynecol Oncol. 2005; 96(3):824-829. [PubMed: 15721432] 


\section{Table 1}

Symptoms of hypersensitivity reactions

\begin{tabular}{llll}
\hline Abdominal pain & Chest pain & Hypertension & Urticaria \\
Angioedema & Diaphoresis & Hypotension & \\
Anxiety & Dyspnea & Rash & \\
Back pain & Flushing & Tachycardia & \\
\hline
\end{tabular}

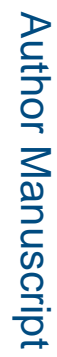

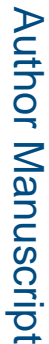

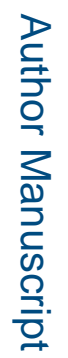


Table 2

Patient characteristics

\begin{tabular}{llll} 
& Group & & \multirow{2}{*}{$\boldsymbol{p}$ value $^{\boldsymbol{a}}$} \\
\cline { 2 - 3 } Characteristic & $\begin{array}{l}\text { Eligible } \\
\boldsymbol{n}(\boldsymbol{\%})\end{array}$ & $\begin{array}{l}\text { Ineligible } \\
\boldsymbol{n}(\%)\end{array}$ & \\
\hline Age in years, mean (SD) & $54.1(11.6)$ & $55(10.9)$ & 0.390 \\
Weight in kilograms, mean (SD) & $78.3(19.3)$ & $80(21.5)$ & 0.379 \\
Female, $N(\%)$ & $232(99.1)$ & $215(100)$ & \\
Male, $N(\%)$ & $2(0.9)$ & $0(0)$ & \\
Breast carcinoma, $N(\%)$ & $232(99.1)$ & $215(100)$ & \\
Angiosarcoma of the breast, $N(\%)$ & $2(0.9)$ & $0(0)$ & \\
Chronic medical conditions $b$-yes, $N(\%)$ & $164(70.0)$ & $156(72.6)$ & 0.602 \\
Stage IV, $N(\%)$ & $28(12.0)$ & $44(20.6)$ & 0.015 \\
\hline
\end{tabular}

$S D$ standard deviation

$a_{p}$ value based on a two-sample $t$ test

${ }^{b}$ One or more of the following: coronary artery disease, hypertension, diabetes, depression, bipolar disorder, generalized anxiety disorder, hyperthyroid, hypothyroid, asthma, COPD, GERD, hyperlipidemia, osteoarthritis, rheumatoid arthritis, lupus, irritable bowel syndrome, fibromyalgia, migraine, cerebral palsy, and colorectal cancer 


\section{Table 3}

\section{Chemotherapy regimens}

\begin{tabular}{lll}
\hline Regimen & $\begin{array}{l}\text { Eligible } \\
\boldsymbol{n}(\boldsymbol{\%})\end{array}$ & $\begin{array}{l}\text { Ineligible } \\
\boldsymbol{n}(\boldsymbol{\%})\end{array}$ \\
\hline Paclitaxel $80 \mathrm{mg} / \mathrm{m}^{2}$ days 1,8 , and $15(21$-day cycle) & $114(48.7)$ & $62(28.8)$ \\
Paclitaxel $80 \mathrm{mg} / \mathrm{m}^{2}+$ trastuzumab days 1,8, and $15(21$-day cycle $)$ & $66(28.2)$ & $35(16.3)$ \\
Paclitaxel $175 \mathrm{mg} / \mathrm{m}^{2}$ day 1 (14-day cycle) & $31(13.2)$ & $63(29.3)$ \\
Paclitaxel $80 \mathrm{mg} / \mathrm{m}^{2}$ days 1,8, and $15(28$-day cycle $)$ & $10(4.3)$ & $18(8.4)$ \\
Paclitaxel $80 \mathrm{mg} / \mathrm{m}^{2}+$ lapatinib + trastuzumab days 1,8, and $15(21$-day cycle $)$ & $5(2.1)$ & $0(0)$ \\
Paclitaxel $90 \mathrm{mg} / \mathrm{m}^{2}+$ bevacizumab days 1,8, and $15(28$-day cycle $)$ & $4(1.7)$ & $7(3.3)$ \\
Paclitaxel $80 \mathrm{mg} / \mathrm{m}^{2}+$ trastuzumab days 1,8, and $15(28$-day cycle $)$ & $2(0.85)$ & $5(2.3)$ \\
Paclitaxel $80 \mathrm{mg} / \mathrm{m}^{2}$ days 1 and 8 (21-day cycle $)$ & $1(0.43)$ & $0(0)$ \\
Paclitaxel $80 \mathrm{mg} / \mathrm{m}^{2}+$ lapatinib days 1,8, and $15(21$-day cycle $)$ & $1(0.43)$ & $0(0)$ \\
Paclitaxel $+\mathrm{carboplatin}$ & $0(0)$ & $20(9.3)$ \\
Paclitaxel $+\mathrm{investigational} \mathrm{agent}$ & $0(0)$ & $3(1.4)$ \\
Paclitaxel $175 \mathrm{mg} / \mathrm{m}^{2}+$ gemcitabine day $1(21$-day cycle $)$ & $0(0)$ & $1(0.47)$ \\
\hline
\end{tabular}




\section{Table 4}

Reasons for ineligibility $(n=215)$

\begin{tabular}{lc}
\hline Characteristic & $\boldsymbol{N}(\%)$ \\
\hline Premedications not removed & $143(66.5)$ \\
Premedications resumed after previously discontinued & $31(14.4)$ \\
Carboplatin or cisplatin & $20(9.3)$ \\
Received previous doses of taxane in 12 months & $7(3.3)$ \\
Switched to different taxane before receiving four doses of paclitaxel & $10(4.7)$ \\
Other $^{a}$ & $4(1.9)$ \\
\hline
\end{tabular}

${ }^{a}$ Patients who did not receive at least two doses of paclitaxel with premedication; patients who had their premedications discontinued after the second paclitaxel dose but did not receive at least two subsequent paclitaxel doses without premedication 